American Journal of Biostatistics 1 (2): 75-81, 2010

ISSN 1948-9889

(C) 2010 Science Publications

\title{
A Novel Technique for Extraction Foetal Electrocardiogram using Adaptive Filtering and Simple Genetic Algorithm
}

\author{
Mahmoud Ahmed Suliman Ali and XiaoPing Zeng \\ Department of Communication, College of Communications Engineering, \\ Chongqing University, P.O. Box 400044, \\ 174shazheng St. Shapingba District, Chongqing, P.R. China
}

\begin{abstract}
Problem statement: Foetal electrocardiogram (FECG) was the best method used to diagnose Foetal heart problem. Knowledge of the foetal heart signal prevents Foetal problems in the earlier stage. Recently, there has been a growing interest in noninvasive method rather than the old invasive method which was more risky for the mother's health. The most significant problem in noninvasive method is the extraction of the Foetal signals from maternal signals and many contaminated noises. The problems of extraction of the Foetal signals are the problems that plagued researchers in the field of signal processing. Objective to develop a technique for extracting FECG signals based on adaptive filter and simple Genetic algorithm. Approach: Practical method for extraction using computer simulations was proposed. The proposed method detects Foetal ECG by denoising abdominal ECG (AECG) and lead to the subsequent cancellation of maternal ECG (MECG) by adaptive filtering. The thoracic signal (TECG) which is purely of Mother signal (MECG) was used to cancel MECG in abdominal signal and the Foetal ECG detector extracts the FECG through Simple Genetic algorithm which enters as the editor of unwanted noise. Results: The FECG signal which was obtained appears to agree with the standard Foetal ECG signals. A program for carrying out the calculations was developed in matlab. The testing of the algorithms was done by using real data from SISTA/DAISY and Physionet. Conclusion: The proposed technique for extraction of FECG was useful and the results appear to agree with the mean values of FECG.
\end{abstract}

Key words: Abdominal electrocardiogram (AECG), Thoracic electrocardiogram (TECG), Genetic Algorithm (GA), surface potentials, processing communities, cutoff frequency, Foetal electrocardiogram (FECG), heartbeat monitoring, interference cancellation, original signal distortion, cardiac activity

\section{INTRODUCTION}

Foetal Electrocardiogram (FECG) extraction is an interesting but a difficult problem in the field of biomedical signal processing. It's a technique for obtaining important information about the condition of the foetal during pregnancy by measuring the electrical signals generated by the foetal heart as measured from multi-channel electrodes placed on the mother's body surface.

Perhaps comes to mind the question, what is the importance of studying the FECG? The answer of that the heart comes the most important member in the body of the animal and if it stops the heart for a few seconds that the animal would lose his life and cut off his work in this world, it can't retrieve an object from the life of the animal ever. It is imperative to detect the importance of heart problems before it's too late. Classified as, heart disease was the most dangerous to human life, of all diseases in the world (Al-Attar, 2010). The importance of understanding cardiac electrophysiology is basic to all clinicians whether in postnatal (pediatric or adult) or prenatal (foetal) medicine. Development in the latter for screening and monitoring purposes is not only an act of preventive medicine, but allows further progress in understanding the foetus as a patient (Velayo et al., 2011).

The problem is how to diagnoses the foetal life in mother abdominal. The most common symptom of foetal death in most of the cases is the decrease in foetal movement which is only diagnosed by examining the absence of cardiac activity in foetal's heart.

Two techniques Doctors using in clinic today to detect the foetal heartbeat:

Corresponding Author: M.A.S. Ali, College of Communications Engineering, Chongqing University, P.O. Box 400044, 174shazheng St. Shapingba District, Chongqing, P.R. China Tel:008613648330442 
- Ultrasound

- Foetal Electrocardiography (FECG)

Ultrasound is weak in detection of foetal's health c onditions compared to FECG (Keralapura et al., 2011). Ultrasound provides only the images but does not confirm that the foetus is alive or dead, especially as the foetus sometimes fall asleep for long periods (A. Poblano et al., 2008). Furthermore, ultrasound techniques require a trained technician/physician, frequent repositioning of the transducer and cannot be done in a home environment - something beneficial for problem pregnancies. Foetal Electrocardiography (FECG) can be an attractive candidate to measure heart conduction signals and is obtained by means of ordinary electrodes placed on the mother's abdomen .

This is where baby heart beat monitored by FECG play a big role. The baby heart beat monitoring is very popular among parents. This doe's not only helps to hear the baby's heart beat but also detect any kind of problem that can prove fatal. Then we can say:

- The baby heart beat monitor helps to detect any kind of problems by constantly monitoring the foetal heartbeat. Not only you get to hear the heartbeat of your baby but also know if there is any kind of abnormality in the baby's condition. The baby's heart beat monitoring is thus of great importance to protect the foetal from dying

- If any kind of abnormality is detected, you can take immediate steps

- It's not only the baby who always benefits from the baby heartbeat monitoring but also the mother's health protected. In case, if the baby's condition goes bad, mother's life is also at risk. So, using baby heartbeat monitor can keep family members alert about the health of the yet to be born and the mother as well

It is very well proved that with the use of baby heartbeat monitor, both the mother and the baby benefits well and that can lead to a successful pregnancy.

The extraction of FECG is one of the goals of biomedical signal processing; as it can remove the cardiac interference and minimizes noise without original signal distortion.

Historically it was first observed Cremer. The early works in this area were done by using galvanometric apparatus of that time, which were limited by the very low amplitude of the foetal signals. As measurement and amplification techniques improved, foetal electrocardiography became more feasible and popular. The limiting factor was then the low foetal SNR, especially in the presence of the strong maternal cardiac interference; a problem which exists up to now. A few decades later, with the developments in computer science and signal processing techniques, automatic signal processing and adaptive filtering techniques were used for foetal R-wave detection (Farvet, 1968) and maternal cardiac interference cancellation. The issue has since been considered as a challenging problem for both biomedical and signal processing communities.

The pioneer researcher used many algorithms for extraction of FECG but a few of them appear met requirement of FECG signals. In this study we will give way can contribute to solving the dilemma of extracting FECG.

Recording of the foetal ECG: There are two methods of recording Foetal ECG (FECG). The first one relies on placing an electrode in direct contact with the scalp of the foetal. This is named an invasive technique that can only be used during labor. The second method of FECG recording is non-invasive and involves attaching electrodes on the maternal abdomen. The signals recorded by invasive methods have better quality as compared with noninvasive methods; but the procedure is rather inconvenient and it's limited to recordings during labor (Sameni et al., 2007). For this reasons the procedure for obtaining the FECG should be noninvasive. The foetal heart is a small heart so that the electrical current it generates is very low. In order to record the FECG, electrodes are placed on the maternal abdomen as close as possible to the foetal heart. The FECG may be acquired by placing a number of electrodes around the general area of the foetal and hoping that at least one of the electrodes will have the FECG with high enough SNR. Beside the problem of electrode placement, noise from electromyography activity affects the signal due to the foetal low voltage signal. Another interfering signal is the maternal ECG (MECG) which can be 5-1000 times higher in its intensity and ability to induce surface potentials (Adam and Shavit, 1990). The MECG effects on all the electrodes placed on the chest (thoracic electrodes) and also effect on the abdominal electrodes, when FECG which effect only on abdominal electrodes.

Foetal signals: The Foetal Electrocardiogram (FECG) is a time-varying signal reflecting the ionic current flow which causes the cardiac fibers to contract and subsequently relax (Malmivuo and Plonsey, 1995). The surface FECG is obtained by recording the potential 
difference between two electrodes placed on the surface of the skin. The standard FECG signal consists of six peak signals each defined with a different letter, the $\mathrm{P}$, $\mathrm{Q}, \mathrm{R}, \mathrm{S}, \mathrm{T}$ and $\mathrm{U}$ peaks. Where the $\mathrm{P}$ peak results from the depolarization of the atrial, the P-R interval is the time between the depolarization of the atria and the depolarization of the ventricles. The QRS-complex results from the depolarization of the ventricles, The $\mathrm{T}$ wave displays the depolarization of the ventricles and the $\mathrm{U}$ wave is usually not present or not important resulting from a rest potential. The origin of the $U$ wave is not clear but it probably represents "after depolarization's" in the ventricles (Lenssen, 2008). The FECG may be divided into the following sections.

P-wave: A small low-voltage deflection away from the Baseline caused by the depolarization of the atria prior to atrial contraction as the activation (depolarization) wave front propagates from the SA node through the atria.

PQ-interval: The time between the beginning of atria depolarization and the beginning of ventricular depolarization.

QRS-complex: The largest-amplitude portion of the FECG caused by currents generated when the ventricles depolarize prior to their contraction. Although atrial repolarization occurs before ventricular depolarization, the latter waveform (i.e., the QRS-complex) is of much greater amplitude and atrial repolarization is therefore not seen on the FECG.

QT-interval: The time between the onset of ventricular depolarization and the end of ventricular repolarization. Clinical studies have demonstrated that the QT-interval increases linearly as the RR-interval increases (Malmivuo and Plonsey, 1995). Prolonged QT-interval may be associated with delayed ventricular repolarization which may cause ventricular tachyarrhythmia's leading to sudden cardiac death.
ST-interval: The time between the end of S-wave and the beginning of T-wave. Significantly elevated or depressed amplitudes away from the baseline are often associated with cardiac illness.

T-wave: Ventricular repolarization, whereby the cardiac muscle is prepared for the next cycle of the ECG.

The signals shape in FECG are the same as at maternal ECG (MECG), but completely different in values. Table 1 shows the variation of the ECG signals value between the maternal and foetal. Figure 1 shows the standard P, Q, R, S, T and U complex signals, the entire non-invasive FECG signal should be processed to be like this shape and around the values shown in Table 1.

We can compare also the maternal normal heart beat rate 72 beat min this as adult heart beat rate and the mean of foetal heart beat rate along gestational period about 120 beat $\min ^{-1}$. There is a variation in heart beat rate because more QRS signal in FECG than QRS signal in MECG.

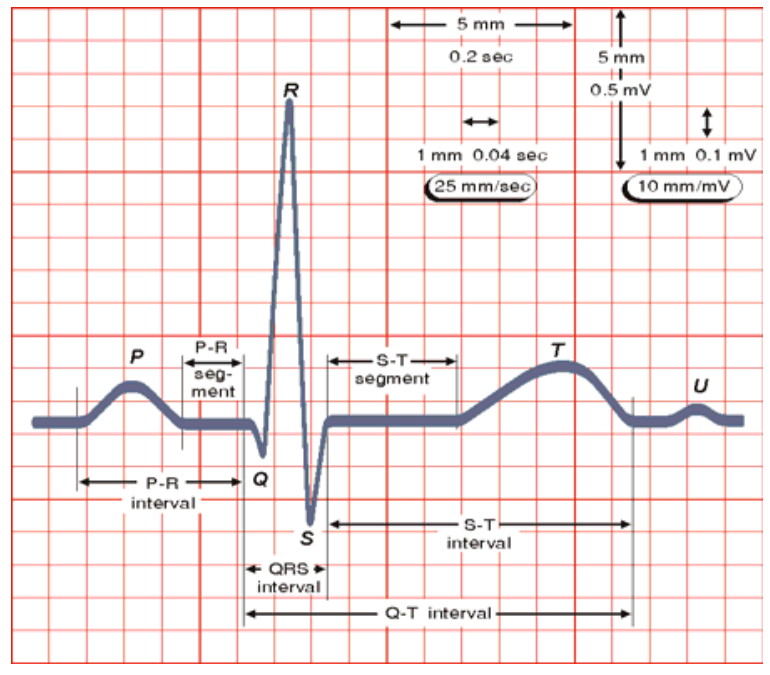

Fig. 1: Standard P, Q, R, S, T and U wave form of human heart; adopted from (Adam and Shavit, 1990)

Table 1: Amplitude-time relations in maternal and foetal electrocardiography signal calculated as mean values from 20 traces recorded between week 36 and 41 of gestation (620 averaged P-QRS-T complexes from maternal and 760 from foetal electrocardiogram were analyzed) (Matonia et al., 2005)

\begin{tabular}{lllllllll}
\hline & $\begin{array}{l}\text { QRS } \\
\text { Amplitude } \\
(\mu \mathrm{v})\end{array}$ & $\begin{array}{l}\text { QRS } \\
\text { width } \\
(\mathrm{ms})\end{array}$ & $\begin{array}{l}\text { PQ } \\
\text { segment } \\
(\mathrm{ms})\end{array}$ & $\begin{array}{l}\text { P } \\
\text { amplitude } \\
(\% \mathrm{QRS})\end{array}$ & $\begin{array}{l}\text { P } \\
\text { width } \\
(\mathrm{ms})\end{array}$ & $\begin{array}{l}\text { ST } \\
\text { segment } \\
(\mathrm{ms})\end{array}$ & $\begin{array}{l}\text { T } \\
\text { amplitude } \\
(\% Q R S)\end{array}$ & $\begin{array}{l}\text { T } \\
\text { width } \\
(\mathrm{ms})\end{array}$ \\
\hline Mother & 150 & 100 & 70 & 20 & 65 & 110 & 30 & 160 \\
Foetal & 30 & 50 & 45 & 10 & 56 & 70 & 25 & 130 \\
\hline
\end{tabular}




\section{MATERIALS AND METHODS}

Extraction model: In this study there are two models, presented the model for signal taken by Thoracic Electrodes (TECG) represented by $\mathrm{x}_{\mathrm{T}}(\mathrm{t})$ and the model for signal taken by Abdominal Electrodes (AECG) represented by $\mathrm{x}_{\mathrm{A}}(\mathrm{t})$ :

$\mathrm{x}_{\mathrm{A}}(\mathrm{t})=\mathrm{M}_{\mathrm{a}}(\mathrm{t})+\mathrm{F}_{\mathrm{a}}(\mathrm{t})+\mathrm{N}_{\mathrm{a}}(\mathrm{t})+\eta_{\mathrm{a}}(\mathrm{t})$

$x_{T}(t)=M_{b}(t)+N_{b}(t)+\eta_{b}(t)$

Where:

$\mathrm{M}_{\mathrm{a}}(\mathrm{t})$ and $\mathrm{M}_{\mathrm{b}}(\mathrm{t})=$ Pure maternal $\mathrm{ECG}$,

$\mathrm{F}_{\mathrm{a}}(\mathrm{t}) \quad=$ A pure foetal ECG

$\eta_{\mathrm{a}}(\mathrm{t}) \quad=\eta_{\mathrm{b}}$ are low-rank or structured noise representing other biological sources that contaminate the ECG and the

$\mathrm{N}_{\mathrm{a}}(\mathrm{t})$ and $\mathrm{N}_{\mathrm{b}}(\mathrm{t})=$ Full-rank observation noise that Always exist in physiological Measurements

As mentioned above the foetal ECG is very weak among the maternal ECG and noise, for this reason it appeared only in Eq. 1 and neglected in Eq. 2.

By Some simplifications the $\eta_{\mathrm{a}}(\mathrm{t})$ and $\eta_{\mathrm{b}}$ can be eliminated by a high pass filter with cutoff frequency of $2 \mathrm{~Hz}$ therefore (1) and (2) simplify to:

$$
\begin{aligned}
& x_{A}(t)=M_{a}(t)+F_{a}(t)+N_{a}(t) \\
& x_{T}(t)=M_{b}(t)+N_{b}(t)
\end{aligned}
$$

The aim here is to extract pure $F_{a}(t)$ which can be obtained by subtracting Eq. 4 from 3 in these equations $\mathrm{M}_{\mathrm{a}}(\mathrm{t}) \neq \mathrm{M}_{\mathrm{b}}(\mathrm{t})$ and $\mathrm{N}_{\mathrm{a}}(\mathrm{t}) \neq \mathrm{N}_{\mathrm{b}}(\mathrm{t})$, for this reasons use factor $\mathrm{K}$ to equalize (4) then get the equation:

$\mathrm{Kx}_{\mathrm{T}}(\mathrm{t})=\mathrm{KM}_{\mathrm{b}}(\mathrm{t})+\mathrm{KN}_{\mathrm{b}}(\mathrm{t})$

Then subtract Eq. 5 from 3 we get:

$$
\begin{aligned}
{\left[\mathrm{x}_{\mathrm{A}}(\mathrm{t})\right.} & \left.-\mathrm{Kx}_{\mathrm{T}}(\mathrm{t})\right]=\left[\mathrm{M}_{\mathrm{a}}(\mathrm{t})-\mathrm{KM}_{\mathrm{b}}(\mathrm{t})\right]+\mathrm{F}_{\mathrm{a}}(\mathrm{t}) \\
& +\left[\mathrm{N}_{\mathrm{a}}(\mathrm{t})-\mathrm{KN}_{\mathrm{b}}(\mathrm{t})\right]
\end{aligned}
$$

Then pure FECG we can get it from the following equation:

$$
\begin{aligned}
F_{a}(t) & =\left[x_{A}(t)-K x_{T}(t)\right]-\left[M_{a}(t)-K_{b}(t)\right] \\
& -\left[N_{a}(t)-K_{b}(t)\right]
\end{aligned}
$$

After cancelling the maternal signal and noise we get:

$F_{a}(t)=\left[x_{A}(t)-K_{T}(t)\right]$

The output of this equation used as input to simple Genetic Algorithms (GA) for removing any undesired signals.

Model Of adaptive filtering and simple genetic algorithm techniques:

Fig. 2 shows the form of the technique method used to extract the desired signal (FECG). The figure contains the steps of the method, the simple Genetic Algorithms (GAs) used here are adaptive heuristic search algorithm premised on the evolutionary ideas of natural selection and genetic.

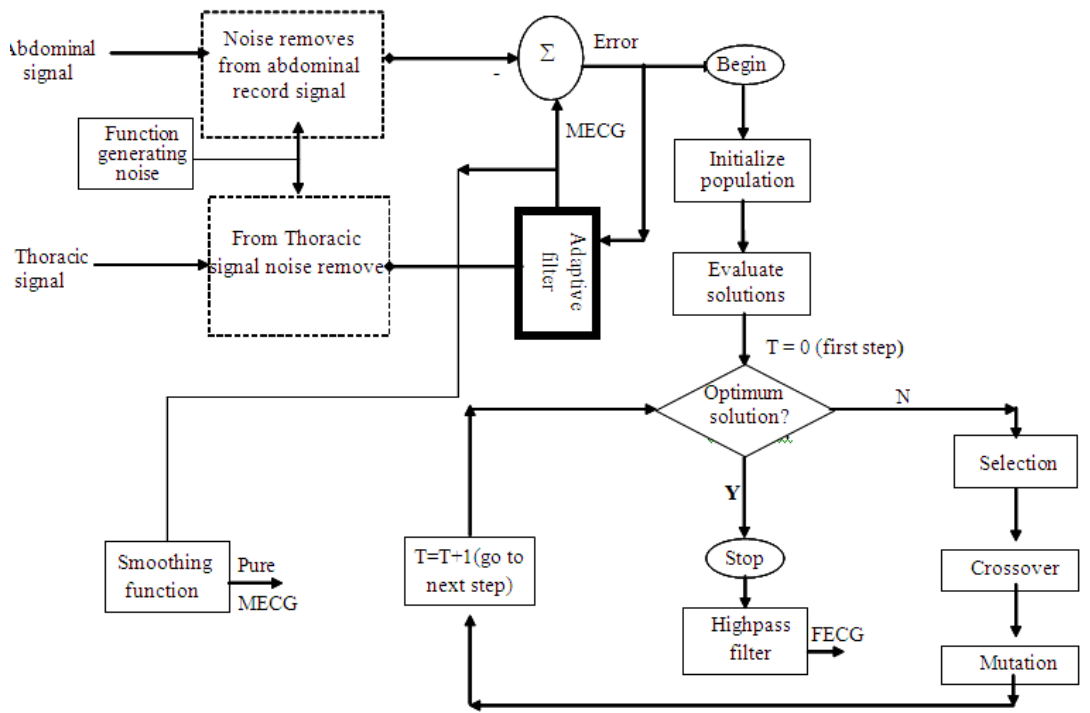

Fig. 2: Shows the step of the form of the method 
Analysis of Maternal Abdominal Signals: Believed that the signals acquired from the wall of abdomen motherland (Non-invasive record) is in fact FECG signal and MECG with several overlapping noise .The analysis of these signals shown in Fig.3 below, the amplitude and frequency range of foetal ECG have been compared with other noises. The labels in this figure stand for the maternal electrocardiogram (mECG), electroencephalogram (mEEG), electrohystrogram (mEHG), electrooculogram (mEOG), electromyogram (mEMG), electrohystrogram (mEHG) and the foetal ECG (fECG).

Adaptive filtering: An adaptive filter is a filter that self-adjusts its transfer function according to an optimization algorithm driven by an error signal. The purpose of an adaptive filter in noise cancellation is to remove the noise from a signal adaptively to improve the signal to noise ratio. The usage of adaptive filters is one of the most popular proposed solutions to reduce the signal corruption caused by predictable and unpredictable noise (Kumar et al., 2010). Adaptive filters are required for some applications because some parameters of the desired processing operation are not known in advance. Adaptive filters have been successfully applied in diverse fields such as communications, radar, seismology, biomedical engineering. Fig .4 shows the diagram of a typical Adaptive Noise Cancellation (ANC) System used for removing noise from human ECG.

In this study used this idea to extract the signal of foetal heart in a better way as contained in Fig. 2.

Genetic algorithm: is a practical method of solving optimization problems on the basis of natural genetics. Genetic algorithm, a powerful \& broadly applicable stochastic research techniques, are the most widely known type of evolutionary computation method today (Kumar et al., 2010). It is also applicable to the problems where traditional estimation and optimization methods are not appropriate (Khodadad and Ardakani, 2008). Genetic algorithms generally start with a population of randomly generated design vectors, test the fitness of those vectors, select the best ones and recombine the parameter values (i.e., exchange some elements) of the best designs. Recently, the genetic algorithms technique was applied to biomedical engineering specially in foetal electrocardiogram signal see (Nazarpour et al., 2007). The architecture see (Fig. 2) used for this study is a combination of an adaptive filter and genetic algorithm (GA), where the

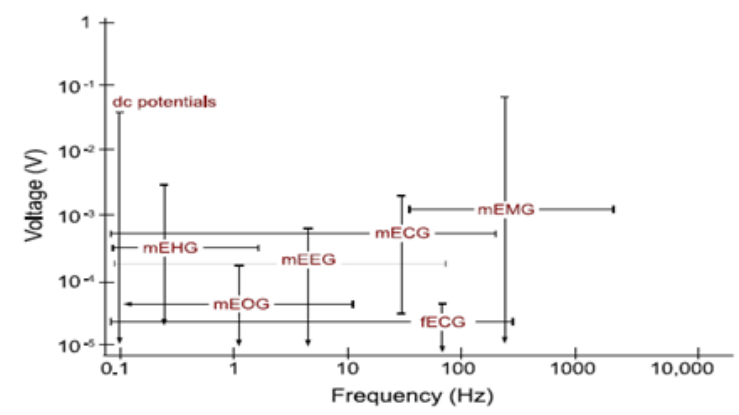

Fig.3: The amplitude and frequency range of biosignals that can interfere with foetal cardiac signals (Sameni and Clifford, 2010)

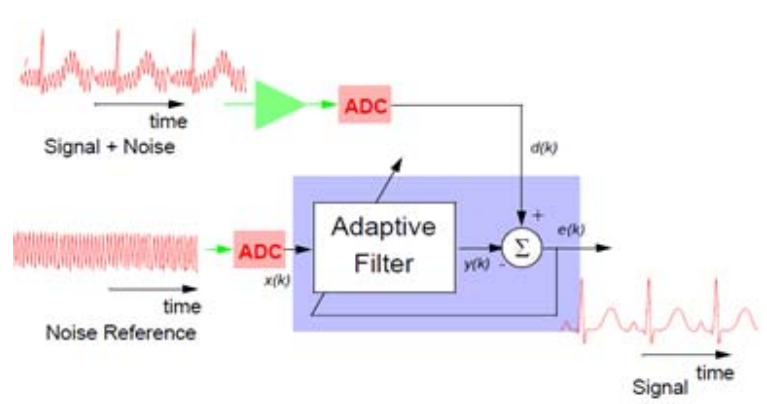

Fig. 4: Adaptive Noise Cancellation (ANC) (Bellanger, 2001)

GA is recruited whenever the first step adaptive filter is suspected of reaching local minima. The second step is an independent GA search without the adaptive filter. The process of exchanging elements among successful designs of GA also has a biological analog, which is referred to as "crossover and mutation". In this study the case was Scheduling problem, so the method used here are string crossover, during crossover step of the algorithm, segment are cut- and - spliced between string. The general framework and basic step of GA can be shown as flowchart appeared at the right part of Fig. 2.

Frequency response and cutoff frequency: It is essential that the ambient noise should be kept as low as possible and this is carried out with the help of an active low pass filter having a cutoff frequency of 70 $\mathrm{Hz}$. This cut off frequency value is selected, because, the foetal heart beats lies in the frequency range of 20 to $70 \mathrm{~Hz}$.(Chourasia and Mittra, 2010).

In this studyr the Filter for Compute frequency response using discrete Fourier transforms (DFT). 


$$
H(z)=\frac{b(1)+b(2) z^{(-1)}+\cdots+b(M+1) z^{(-m)}}{a(1)+a(2) z^{(-1)}+\cdots+a(N+1) z^{(-n)}}
$$

Where :

$$
\begin{array}{ll}
\mathrm{b} & =\text { coefficient vector of numerator polynomial } \\
\mathrm{a} & =\text { coefficient vector of denominator polynomial }
\end{array}
$$

After optimization foetus signal, designed a highpass filter with normalized digital cutoff frequency, $\Omega_{\mathrm{c}}=\omega_{\mathrm{c}} \frac{\mathrm{T}}{\pi}$, where equal $0.1 \mathrm{H}(\mathrm{z})$ and number of poles equal 3 .

FECG database: for testing the algorithm used public databases widely used by the signal processing community known as SISTA/DAISY dataset.

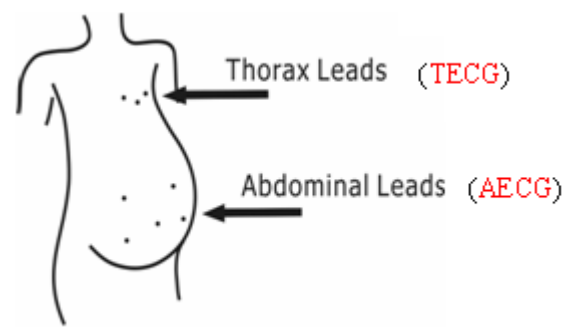

Fig.5: Positions electrodes lead on the body of Mother

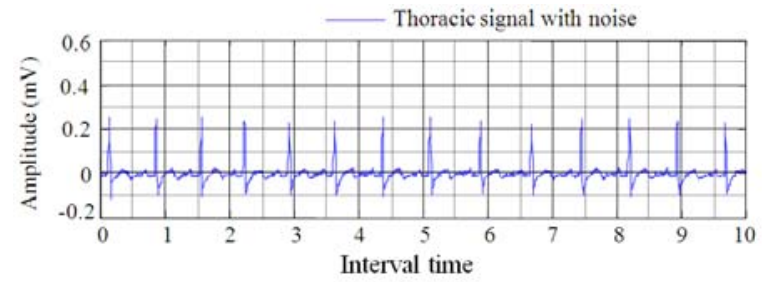

(a)

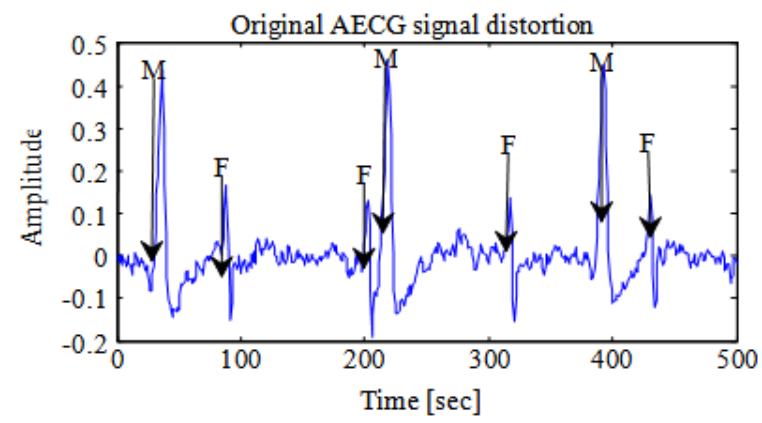

(b)

Fig. 6:(a) show graph for original TECG, (b) show graph for original AECG
Consists of a single dataset of cutaneous potential recording of a pregnant woman. A total of 8 channels (4 abdominal and 3 thoracic) are available, sampled at 500 $\mathrm{Hz}$, lasting 10 seconds and The lengths of the data were 2500 point. This dataset is part of a database know as the SISTA (Signals, Identification, System Theory and Automation) database from the SISTA group of the department of Electrical Engineering of the Katholieke niversiteit Leuven, Belgium.

Believed the locations of leads on maternal body for an 8-channel maternal ECG acquisition system are shown in Fig. 5 below. Maternal thorax ECG (TECG) signals are sampled from thorax leads while maternal abdominal ECG (AECG) signals are obtained from abdominal leads.

These methods can be practically applied already since $11^{\text {th }}$ week of gestation, but the signal quality depends on recording period between $26-41^{\text {th }}$ week of gestation.

Original signal distortion: Fig. 6 below contains two graphs (a) and (b); the (a) graph shows real thoracic signal (TECG) of maternal which contain MECG + noise and the (b) graph shows real sample of abdominal signal (AECG) which contains MECG + FECG + noise. Where $\mathrm{M}$ denoted to maternal QRS amplitude region and $\mathrm{F}$ denoted to foetal QRS amplitude region. These graphs shows direct plot using SISTA/DAISY data without any filter effects.

\section{RESULTS}

The maternal ECG signal interference was canceled from the foetal heart ECG signal .ECG signals are given as an input and is simulated using MATLAB. The result of programs is shown in the Fig. 7 below.
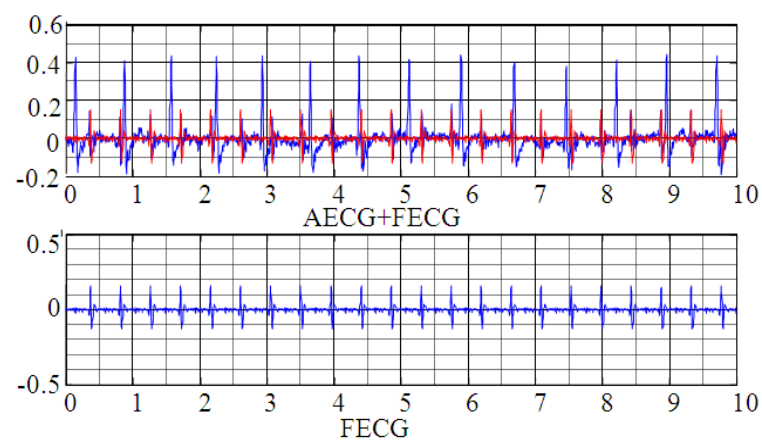

Fig. 7: A graph for MECG and FECG recombined after extracted, B graph for FECG 


\section{DISCUSSION}

Figure 7 in results above contains two graphs the upper graph shows AECG and FECG recombined after being extracted. In comparing these two signals we can see the region of FECG in AECG signal. The lower graph shows pure FECG.

The amplitude of QRS of FECG is about 30 microvolt while that of MECG is about 150 microvolt. These values may agree to the value of QRS amplitude appeared in Table 1. The obtained result shows the effective ness of the proposed algorithm.

\section{CONCLUSION}

In this study we present a Hybrid adaptive filtering simple genetic algorithm for removing undesired signals which are difficult to be removed by normal filters. The algorithm used in this study is very simple and not complex. The performance and validity of the proposed algorithm have been confirmed by computer simulations and experiment on real-world ECG data. The result which was obtained appears to agree with the standard Foetal ECG signals. The Researcher recommends following this method to gain more useful results.

\section{ACKNOWLEDGEMENT}

Many thanks to china CSC for offering scholarship for me and Many thanks to My friends Muhammad Aamir and Al-Jbori A'ssim for their useful comments on the manuscript.

\section{REFERENCES}

Adam, D. and D. Shavit, 1990. Complete foetal ECG morphology recording by synchronised adaptive filtration. Med. Biol. Eng. Comput., 28: 287-292. PMID: 2246925

Al-Attar, A.M., 2010. Physiological study on the effect of Acalypha Wilkesiana leaves extract on streptozotocin-induced experimental diabetes in male mice. Am. Med. J., 1: 51-58. DOI: 10.3844/amjsp.2010.51.58

Bellanger, M., 2001. Adaptive Digital Filters. 2nd Edn., CRC Press, USA., ISBN-10: 0203903846, pp: 464.

Chourasia, V. and A. Mittra, 2010. Passive acoustic signal acquisition system for non-invasive fetal heart sound monitoring applications. Internet $\mathrm{J}$. Med.

Technol.

http://www.ispub.com/journal/the_internet_journal _of_medical_technology/volume_5_number_1_50/ article/passive-acoustic-signal-acquisition-systemfor-non-invasive-fetal-heart-sound-monitoringapplications.html
Farvet, A.G., 1968. Computer matched filter location of fetalR-waves. Med. Biol. Eng., 6: 467-475. DOI: 10.1007/BF02474285

Keralapura, M., M. Pourfathi and B. Sirkeci-Mergen, 2011. Impact of contrast functions in Fast-ICA on twin ECG separation. IAENG Int. J. Comput. Sci., 38: $1-10$.

Khodadad, M. and M.D. Ardakani, 2008. Inclusion identification by inverse application of boundary element method, genetic algorithm and conjugate gradient method. Am. J. Applied Sci., 5: 11581166. DOI: 10.3844/ajassp.2008.1158.1166

Kumar, D.R., E.R. Kumar, E.S. Gill and E.A. Kaushik, 2010. Genetic algorithm approach to operating system process scheduling problem. Int. J. Eng. Sci. Technol., 2: 4247-4252.

Lenssen, A.M.J., 2008. From cardiac electrical activity to the ECG: A finite element model. Master thesis, Eindhoven University of Technology. http://www.mate.tue.nl/mate/pdfs/9185.pdf

Malmivuo, J. and R. Plonsey, 1995. Bioelectromagnetism: Principles and Applications of Bioelectric and Biomagnetic Fields. 1st Edn., Oxford University Press, USA., ISBN-10: 0195058232, pp: 482.

Matonia, A., J. Jeewski, T. Kupka, J. Wrobel and K. Horoba, 2005. Modelling of Non-invasively Recorded Maternal and Fetal Electrocardiographic Signals. Biocybernetics Biomed. Eng., 25: 27-39.

Nazarpour, K., S. Ebadi and S. Sane, 2007. Fetal electrocardiogram signal modelling using genetic algorithm. Proceedings of the International Workshop on Medical Measurements and Applications, May 4-5, IEEE Xplore, Warsaw, pp: 1-4. DOI: 10.1109/MEMEA.2007.4285156

Poblano, A., R. Haro and C. Arteaga, 2008. Neurophysiologic measurement of continuity in the sleep of fetuses during the last week of pregnancy and in newborns. Int. J. Biol. Sci., 4: 23-28. PMID: 18167533

Sameni, R. and G.D. Clifford, 2010. A review of fetal ECG signal processing issues and promising directions, Harvard University, Boston, USA. http://www.robots.ox.ac.uk/ gari/papers/Sameni_C lifford_TOPETJ_10.pdf

Sameni, R., M.B. Shamsollahi, C. Jutten and G.D. Cliord, 2007. A nonlinear bayesian filtering framework for ecg denoising. IEEE Trans. Biomed. Eng., $\quad 54$ : 2172-2185. $\quad$ DOI: 10.1109/TBME.2007.897817

Velayo, C., N. Sato, T. Ito, H. Chisaka and N. Yaegashi et al. 2011. Understanding congenital heart defects through abdominal fetal electrocardiography: Case reports and clinical implications. J. Obstetrics Gynaecol. Res. DOI: $10.1111 / \mathrm{j} .1447-$ 0756.2010.01367.x 